\title{
Wellposedness of the two- and three-dimensional full water wave problem
}

\author{
Sijue $\mathrm{Wu}$
}

\section{Introduction}

The mathematical problem of $n$-dimensional water wave concerns the motion of the interface separating an inviscid, incompressible, irrotational fluid, under the influence of gravity, from a region of zero density (i.e. air) in $n$-dimensional space. It is assumed that the fluid region is below the air region. Assume that the density of the fluid is 1, the gravitational field is $-\mathbf{k}$, where $\mathbf{k}$ is the unit vector pointing in the upward vertical direction, and at time $t \geq 0$, the free interface is $\Sigma(t)$, and the fluid occupies region $\Omega(t)$. When surface tension is zero, the motion of the fluid is described by

$$
\begin{cases}\mathbf{v}_{t}+\mathbf{v} \cdot \nabla \mathbf{v}=-\mathbf{k}-\nabla P & \text { on } \Omega(t), t \geq 0, \\ \operatorname{div} \mathbf{v}=0, \quad \operatorname{curl} \mathbf{v}=0, & \text { on } \Omega(t), t \geq 0, \\ P=0, & \text { on } \Sigma(t) \\ (1, \mathbf{v}) \text { is tangent to the free surface }(t, \Sigma(t)),\end{cases}
$$

where $\mathbf{v}$ is the fluid velocity, $P$ is the fluid pressure. It is well-known that when surface tension is neglected, the water wave motion can be subject to the Taylor instability $[\mathbf{3 0}, \mathbf{3}, \mathbf{2}]$. Assume that the free interface $\Sigma(t)$ is described by $\xi=\xi(\alpha, t)$, where $\alpha \in R^{n-1}$ is the Lagrangian coordinate, i.e. $\xi_{t}(\alpha, t)=\mathbf{v}(\xi(\alpha, t), t)$ is the fluid velocity on the interface, $\xi_{t t}(\alpha, t)=$ $\left(\mathbf{v}_{t}+\mathbf{v} \cdot \nabla \mathbf{v}\right)(\xi(\alpha, t), t)$ is the acceleration. Let $\mathbf{n}$ be the unit normal pointing out of $\Omega(t)$. The Taylor sign condition relating to Taylor instability is

$$
-\frac{\partial P}{\partial \mathbf{n}}=\left(\xi_{t t}+\mathbf{k}\right) \cdot \mathbf{n} \geq c_{0}>0
$$

point-wisely on the interface for some positive constant $c_{0}$. In $[\mathbf{3 2}, \mathbf{3 3}]$, we showed that the Taylor sign condition (1.2) always holds for the $n$-dimensional infinite depth water wave problem (1.1), $n \geq 2$, as long as the interface is non-self-intersecting; and the initial value problem of the

Financial support in part by NSF grant DMS-0800194.

(C) 2011 International Press 
water wave system (1.1) is uniquely solvable locally in time in Sobolev spaces for arbitrary given data. Earlier work include Nalimov [20], Yosihara [36] and Craig [11] on local existence and uniqueness for small data in 2D. Notice that if surface tension is not zero, or if there is a bottom, or nonzero-vorticity, the Taylor sign condition need not hold. Local wellposedness for water wave motion with the effect of surface tension, bottom and a non-zero vorticity, under the assumption $(1.2)$ can be found in $[\mathbf{1}, \mathbf{5}, \mathbf{6}, \mathbf{1 5}, \mathbf{1 8}, \mathbf{1 9}, \mathbf{2 1}, \mathbf{2 5}, \mathbf{3 7}$ ].

To understand the large time behavior of solutions for the water wave problem (1.1), it is necessary to understand the nature of its nonlinearity.

From the fluids literature [12], we know that "wavelengths which are generated in the open ocean can be very large; swell as long as $800 \mathrm{~m}$ between crests has been recorded. The wave heights are limited, the theoretical limit being about one seventh of the wave length. Records of giant waves have to be regarded with some caution, and a survey taken from sailing ships' logs showed that $45 \%$ of waves are less than $1.25 \mathrm{~m}$ in height between trough and crest, with only $10 \%$ greater than $6 \mathrm{~m}$. Nevertheless there is a reliable record of a wave $34 \mathrm{~m}$ in height.

We shall see that waves of different wavelengths travel at different speeds, so that a giant wave maybe produced for a short time by a combination of shorter and longer waves. However, in some parts of the world the occurrence of giant waves is apparently more frequent, for example off the east coast of South Africa, where long waves originating in the 'roaring forties' meet the Agulhas current flowing towards them. Adverse currents amplify waves and can bend them to give a focusing effect. There are numerous reports of ships being damaged by waves in this area. In 1973 two large ships were damaged.... Something similar probably accounted for the disappearance of the Waratah, a liner on only her second voyage, in 1909. No trace of her was ever found, although there had been other ships in the area at the time, including sailing ships, which had not been in difficulties."

Such giant waves is the so called rogue waves. From the above description it seems that rogue waves can appear in perfectly clear weather in open ocean. Conjectures on possible causes include: diffractive focusing due to certain coastal shapes; focusing by currents; and nonlinear effect-such as that of a focusing nonlinear Schrödinger equation.

In our recent work $[\mathbf{3 4}, \mathbf{3 5}]$ we focused on understanding the nonlinear effect. We therefore assumed there is no wind, no coast, no bottom. For large scale waves such as those in open ocean, it is reasonable to neglect surface tension. We studied the behavior of solutions of system (1.1) for large time for data that are small in appropriate senses.

In $[\mathbf{3 4}, \mathbf{3 5}]$, we showed that for the $2 \mathrm{D}$ water wave problem $(1.1)(n=$ $2)$, the quantity $\Theta=(I-\mathfrak{H}) y$, under an appropriate coordinate change $k=k(\alpha, t)$, satisfies an equation of the type

$$
\partial_{t}^{2} \Theta-i \partial_{\alpha} \Theta=\mathcal{G}
$$


with $\mathcal{G}$ consisting of nonlinear terms of only cubic and higher orders. Here $\mathfrak{H}$ is the Hilbert transform related to the water region $\Omega(t), y$ is the height function for the interface $\Sigma(t): \xi=(x(\alpha, t), y(\alpha, t)), \alpha \in \mathbb{R}$. Similarly, for the 3D water wave problem (1.1) $(n=3)$, if $\Sigma(t): \xi=(x(\alpha, \beta, t), y(\alpha, \beta, t)$, $z(\alpha, \beta, t)),(\alpha, \beta) \in \mathbb{R}^{2}$ is the interface at time $t$, and $\mathfrak{H}$ is the Hilbert transform associated to the water region $\Omega(t), N=\xi_{\alpha} \times \xi_{\beta}$ is the outward normal, then the quantity $\theta=(I-\mathfrak{H}) z$ satisfies such an equation

$$
\partial_{t}^{2} \theta-\mathfrak{a} N \times \nabla \theta=G
$$

where $G$ is a nonlinearity of cubic and higher orders in nature. Furthermore we found a coordinate change $k$ that transforms (1.4) into an equation consisting of a linear part plus only cubic and higher order nonlinear terms. Using these favorable structures, we showed that for water waves in two space dimensions, if initially the amplitude of the interface (and finitely many of its derivatives) is of size $O(\epsilon)$ and the kinetic energy is of size $O\left(\epsilon^{2}\right)$ and small, then there exists a unique classical solution of the water wave equation (1.1) $(n=2)$ for a time period $\left[0, e^{c / \epsilon}\right]$; during this time period, the amplitude of the interface remains small and is as regular as the initial interface. Here $c$ is a constant independent of $\epsilon$ (c.f. Theorem 3.8 and [34]). For water waves in three space dimensions, if initially the steepness of the interface and the fluid velocity on the interface (and finitely many of their derivatives) are small, then there exists a unique classical solution of the water wave equation (1.1) $(n=3)$ for all time, and the interface remains to have small steepness and is as regular as the initial interface for all time (c.f. Theorem 3.9 and [35]). We note that in our result for the 3D water waves, no direct assumptions are made to the height of the initial interface and the velocity field in the fluid domain. In particular, the amplitude of the initial interface can be arbitrary large, the initial kinetic energy $\frac{1}{2}\|\mathbf{v}\|_{L^{2}(\Omega(0))}^{2}$ can be infinite. We also note that although our result for the $2 \mathrm{D}$ water waves [34] requires the initial kinetic energy $\frac{1}{2}\|\mathbf{v}\|_{L^{2}(\Omega(0))}^{2}$ being small, while viewed as a special case of $3 \mathrm{D}$ where the wave is constant in one direction, its $3 \mathrm{D}$ energy in fact is infinite $\left(\infty \times \epsilon^{2}=\infty\right)$.

Before moving on to some detailed discussions, we mention the main idea in proving the Taylor sign condition (1.2) for the water wave equation (1.1):

Applying div to both sides of the Euler equation, and using the assumption that $\operatorname{curl} \mathbf{v}=0$, we find

$$
\Delta P=-|\nabla \mathbf{v}|^{2} \leq 0 \quad \text { in } \Omega(t) .
$$

Therefore from $P=0$ on the interface $\Sigma(t)$ and the maximal principle, we have $-\frac{\partial P}{\partial \mathbf{n}} \geq 0$. For a precise proof of the fact that the Taylor sign condition (1.2) always holds, we refer to $[\mathbf{3 2}, \mathbf{3 3}]$.

We now give some details on what we have obtained so far (c.f. [32, 33, 34, 35]). Notice that equation (1.1) is a nonlinear equation defined in moving domains. It is difficult to obtain results directly from it. One key 
step in our approach is to rewrite (1.1) into forms from which results and informations can be obtained.

\section{Local wellposedness of the $2 \mathrm{D}$ and $3 \mathrm{D}$ water wave problems}

We first discuss some of the main ideas in proving the local in time well-posedness of the water wave system $(1.1)[\mathbf{3 2}, \mathbf{3 3}]$. For clarity, we mainly write in terms of the $2 \mathrm{D}$ water wave, we will only mention the main differences and ideas for 3D.

Let $\xi=\xi(\alpha, t)=(x(\alpha, t), y(\alpha, t)), \alpha \in \mathbb{R}$, be the equation of the free interface $\Sigma(t)$ at time $t$ in Lagrangian coordinates $\alpha$. In what follows, we regard the 2-D space as a complex plane and use the same notation for a complex form $\xi=x+i y$ and a point $\xi=(x, y)$. So $\bar{\xi}=x-i y$. We write $[A, B]=A B-B A$.

As we know it is difficult to solve (1.1) directly since it is defined on moving domains. The first step used in $[\mathbf{3 2}, \mathbf{3 3}]$ in solving (1.1) was to reduce it to an equivalent equation defined on the moving interface $\Sigma(t){ }^{1}$ This was done based on the following observations. First notice that the second equation in (1.1): $\operatorname{div} \mathbf{v}=0, \operatorname{curl} \mathbf{v}=0$ implies that the complex conjugate of the velocity field $\overline{\mathbf{v}}$ is holomorphic in $\Omega(t)$. Therefore the trace of the velocity on $\Sigma(t): \xi_{t}(\cdot, t)=\mathbf{v}(\xi(\cdot, t), t)$ satisfies $\bar{\xi}_{t}=\mathfrak{H} \bar{\xi}_{t}$, where

$$
\mathfrak{H} f(\alpha, t)=\frac{1}{\pi i} p \cdot v \cdot \int \frac{f(\beta, t) \xi_{\beta}(\beta, t)}{\xi(\alpha, t)-\xi(\beta, t)} d \beta
$$

is the Hilbert transform on $\Sigma(t)$ associated with the parameterization $\xi=\xi(\alpha, t)$. Now from the third equation in (1.1): $P=0$ on $\Sigma(t)$, we know $\nabla P$ points in the direction normal to $\Sigma(t)$, therefore $-\nabla P=i \mathfrak{a} \xi_{\alpha}$, with $\mathfrak{a}=-\frac{\partial P}{\partial \mathbf{n}} \frac{1}{\left|\xi_{\alpha}\right|}$. So the trace of the Euler equation on $\Sigma(t)$ can be written as $\xi_{t t}+i=i \mathfrak{a} \xi_{\alpha}$, and the system (1.1) is equivalent to the following system on the interface $\Sigma(t)$ :

$$
\left\{\begin{array}{l}
\xi_{t t}+i=i \mathfrak{a} \xi_{\alpha} \\
\bar{\xi}_{t}=\mathfrak{H} \bar{\xi}_{t}
\end{array}\right.
$$

However (2.2) is fully nonlinear. To solve (2.2), we further reduced it into a quasilinear equation $[\mathbf{3 2}, \mathbf{3 3}]$, so that on which the classical energy method could be applied. This was done by taking one derivative to $t$ to the first equation in (2.2). Taking derivative to $t$ to the first equation in (2.2), we got

$$
\left\{\begin{array}{l}
\xi_{t t t}-i \mathfrak{a} \xi_{t \alpha}=i \mathfrak{a}_{t} \xi_{\alpha} \\
\bar{\xi}_{t}=\mathfrak{H} \bar{\xi}_{t}
\end{array}\right.
$$

\footnotetext{
${ }^{1}$ The approaches of Nalimov [20], Yosihara [36] and Craig [11] were also to reduce the system (1.1), for $n=2$ only, to a system defined on the moving interface.
} 
Using the fact that $\bar{\xi}_{t}=\mathfrak{H} \bar{\xi}_{t}$, we deduced

$$
\begin{aligned}
(I & -\mathfrak{H})\left(-i \mathfrak{a}_{t} \bar{\xi}_{\alpha}\right)=(I-\mathfrak{H})\left(\bar{\xi}_{t t t}+i \mathfrak{a} \bar{\xi}_{t \alpha}\right) \\
& =\left[\partial_{t}^{2}+i \mathfrak{a} \partial_{\alpha}, \mathfrak{H}\right] \bar{\xi}_{t} \\
& =2\left[\xi_{t t}, \mathfrak{H}\right] \frac{\bar{\xi}_{t \alpha}}{\xi_{\alpha}}+2\left[\xi_{t}, \mathfrak{H}\right] \frac{\bar{\xi}_{t t \alpha}}{\xi_{\alpha}}-\frac{1}{\pi i} \int\left(\frac{\xi_{t}(\alpha, t)-\xi_{t}(\beta, t)}{\xi(\alpha, t)-\xi(\beta, t)}\right)^{2} \bar{\xi}_{t \beta} d \beta
\end{aligned}
$$

Further using the fact that $\mathfrak{a}$ and $\mathfrak{a}_{t}$ are real valued gave

$$
\begin{aligned}
& \left(I+\mathfrak{K}^{*}\right)\left(\mathfrak{a}_{t}\left|\xi_{\alpha}\right|\right) \\
& \quad=\operatorname{Re}\left(\frac{i \xi_{\alpha}}{\left|\xi_{\alpha}\right|}\left\{2\left[\xi_{t t}, \mathfrak{H}\right] \frac{\bar{\xi}_{t \alpha}}{\xi_{\alpha}}+2\left[\xi_{t}, \mathfrak{H}\right] \frac{\bar{\xi}_{t t \alpha}}{\xi_{\alpha}}-\frac{1}{\pi i} \int\left(\frac{\xi_{t}(\alpha, t)-\xi_{t}(\beta, t)}{\xi(\alpha, t)-\xi(\beta, t)}\right)^{2} \bar{\xi}_{t \beta} d \beta\right\}\right)
\end{aligned}
$$

here $R e \xi$ indicates the real part of $\xi$,

$$
\mathfrak{K}^{*} f(\alpha, t)=p . v \cdot \int \operatorname{Re}\left\{\frac{-1}{\pi i} \frac{\xi_{\alpha}}{\left|\xi_{\alpha}\right|} \frac{\left|\xi_{\beta}(\beta, t)\right|}{(\xi(\alpha, t)-\xi(\beta, t))}\right\} f(\beta, t) d \beta
$$

is the adjoint of the double layered potential operator $\mathfrak{K}$ in $L^{2}(\Sigma(t), d S)$. Notice that $I+\mathfrak{K}^{*}$ is invertible on $L^{2}(\Sigma(t), d S)$. From (2.5) we see that $\mathfrak{a}_{t}\left|\xi_{\alpha}\right|$ has the same regularity as that of $\bar{\xi}_{t t}$ and $\bar{\xi}_{t}$.

We now rewrite $(2.3)$ as

$$
\left\{\begin{array}{l}
\xi_{t t t}-i \mathfrak{a} \xi_{t \alpha}=\frac{\xi_{t t}+i}{\left|\xi_{t t}+i\right|} \mathfrak{a}_{t}\left|\xi_{\alpha}\right| \\
\bar{\xi}_{t}=\mathfrak{H} \bar{\xi}_{t}
\end{array}\right.
$$

and use (2.5) for $\mathfrak{a}_{t}\left|\xi_{\alpha}\right|$. Notice that $i \partial_{\alpha} \xi_{t}=-\nabla_{\mathbf{n}} \xi_{t}$, and the DirichletNeumann operator $\nabla_{\mathbf{n}}$ is a positive operator. As we have seen the Taylor sign condition $(1.2)$ holds, i.e. $\mathfrak{a}>0[\mathbf{3 2}, \mathbf{3 3}]$. Therefore $(2.6)$ is a weakly hyperbolic quasi-linear system with the right hand side of the first equation in (2.6) consisting of terms of lower order derivatives of $\xi_{t}$. The local in time wellposedness of (2.6)-(2.5) in Sobolev spaces (with $\left(\xi_{t}, \xi_{t t}\right) \in$ $\left.C\left([0, T], H^{s+1 / 2} \times H^{s}\right)\right)$ was then proved by energy estimates and a fixed point iteration argument. Through establishing the equivalence of (1.1) with (2.6)-(2.5), we obtained the local in time well-posedness in Sobolev spaces of the full water wave equation (1.1). For details of the proof, we refer the readers to $[\mathbf{3 2}, \mathbf{3 3}]$.

We now mention some of the main ideas in deriving the quasi-linear system for the $3 \mathrm{D}$ water waves. To derive a similar equivalent quasilinear system for the $3 \mathrm{D}$ water wave equation (1.1) $(n=3)$, naturally we looked for a suitable counterpart in 3D of the equation for the trace on the interface of "div $\mathbf{v}=0, \operatorname{curl} \mathbf{v}=0$ ". We found Clifford analysis offers a suitable framework for 3D water waves. Let's recall the basics of Clifford algebra, or in other words, the algebra of quaternions $\mathcal{C}\left(V_{2}\right)$ (c.f. [14]). Let $\left\{1, e_{1}, e_{2}, e_{3}\right\}$ 
be the basis of $\mathcal{C}\left(V_{2}\right)$, satisfying

$$
e_{i}^{2}=-1, \quad \text { for } i=1,2,3, \quad e_{i} e_{j}=-e_{j} e_{i}, \quad i \neq j, \quad e_{3}=e_{1} e_{2} .
$$

Let $\mathcal{D}=\partial_{x} e_{1}+\partial_{y} e_{2}+\partial_{z} e_{3}$. By definition, a Clifford-valued function $F$ : $\Omega \subset \mathbb{R}^{3} \rightarrow \mathcal{C}\left(V_{2}\right)$ is Clifford analytic in domain $\Omega$ if $\mathcal{D} F=0$ in $\Omega$. Therefore $F=\sum_{i=1}^{3} f_{i} e_{i}$ is Clifford analytic in $\Omega$ if and only if $\operatorname{div} F=0$ and $\operatorname{curl} F=0$ in $\Omega$. Furthermore we know Clifford analytic functions have the property that $F$ is Clifford analytic in $\Omega$ if and only if $F=\mathfrak{H}_{\Sigma} F$, where

$$
\mathfrak{H}_{\Sigma} g(\alpha, \beta)=p . v \cdot \iint K\left(\eta\left(\alpha^{\prime}, \beta^{\prime}\right)-\eta(\alpha, \beta)\right)\left(\eta_{\alpha^{\prime}}^{\prime} \times \eta_{\beta^{\prime}}^{\prime}\right) g\left(\alpha^{\prime}, \beta^{\prime}\right) d \alpha^{\prime} d \beta^{\prime}
$$

is the $3 \mathrm{D}$ version of the Hilbert transform on $\Sigma=\partial \Omega: \eta=\eta(\alpha, \beta),(\alpha, \beta) \in$ $\mathbb{R}^{2}$, with normal $\eta_{\alpha} \times \eta_{\beta}$ pointing out of $\Omega$, and

$$
\Gamma(\eta)=-\frac{1}{\omega_{3}|\eta|}, \quad K(\eta)=-2 \mathcal{D} \Gamma(\eta)=-\frac{2}{\omega_{3}} \frac{\eta}{|\eta|^{3}},
$$

$\omega_{3}$ is the surface area of the unit sphere in $\mathbb{R}^{3}$.

Therefore if $\xi=\xi(\alpha, \beta, t),(\alpha, \beta) \in \mathbb{R}^{2}$ is the free interface $\Sigma(t)$ in Lagrangian coordinates $(\alpha, \beta)$ at time $t$, with $N=\xi_{\alpha} \times \xi_{\beta}$ pointing out of the fluid domain, we can rewrite the $3 \mathrm{D}$ water wave system (1.1) $(n=3)$ as (c.f. $[33])$

$$
\left\{\begin{array}{l}
\xi_{t t}+e_{3}=\mathfrak{a} N \\
\xi_{t}=\mathfrak{H}_{\Sigma(t)} \xi_{t}
\end{array}\right.
$$

where $\mathfrak{a}=-\frac{1}{|N|} \frac{\partial P}{\partial \mathbf{n}}$. From the nonlinear system (2.9) we derived the quasilinear system (cf. (5.21)-(5.22) of [33]) for the 3D water waves, and the local in time well-posedness of the $3 \mathrm{D}$ full water wave system was therefore obtained from energy estimates and a fixed point iteration argument to the quasi-linear system [33].

Before ending this section, we point out that the quasilinear system (2.6)(2.5) (and (5.21)-(5.22) of [33] for 3D water waves) is coordinate invariant. Let $U_{g} f(\alpha, t)=f \circ g(\alpha, t)=f(g(\alpha, t), t)$. For fixed $t$, let $k=k(\alpha, t)$ : $\mathbb{R} \rightarrow \mathbb{R}$ be a diffeomorphism and $k_{\alpha}>0$. Let $k^{-1}$ be such that $k \circ k^{-1}(\alpha, t)=$ $\alpha$. Define

$$
\zeta=\xi \circ k^{-1}, \quad b=k_{t} \circ k^{-1} \quad \text { and } \quad A \circ k=\mathfrak{a} k_{\alpha}
$$

Let

$$
D_{t}=U_{k}^{-1} \partial_{t} U_{k}=\partial_{t}+b \partial_{\alpha}
$$

be the material derivative. By a simple application of the chain rule, we have

$$
U_{k}^{-1}\left(\partial_{t}^{2}-i \mathfrak{a} \partial_{\alpha}\right) U_{k}=D_{t}^{2}-i A \partial_{\alpha},
$$


and equation (2.6) becomes

$$
\left\{\begin{array}{l}
\left(D_{t}^{2}-i A \partial_{\alpha}\right) D_{t} \zeta=\left(\mathfrak{a}_{t}\left|\xi_{\alpha}\right|\right) \circ k^{-1} \frac{D_{t}^{2} \zeta+i}{\left|D_{t}^{2} \zeta+i\right|} \\
\overline{D_{t} \zeta}=\mathcal{H} \overline{D_{t} \zeta}
\end{array}\right.
$$

with

$$
\begin{aligned}
(I+ & \left.\mathcal{K}^{*}\right)\left(\left(\mathfrak{a}_{t}\left|\xi_{\alpha}\right|\right) \circ k^{-1}\right) \\
= & \operatorname{Re}\left(\frac { i \zeta _ { \alpha } } { | \zeta _ { \alpha } | } \left\{2\left[D_{t}^{2} \zeta, \mathcal{H}\right] \frac{\partial_{\alpha} \overline{D_{t} \zeta}}{\zeta_{\alpha}}+2\left[D_{t} \zeta, \mathcal{H}\right] \frac{\partial_{\alpha} \overline{D_{t}^{2} \zeta}}{\zeta_{\alpha}}\right.\right. \\
& \left.\left.-\frac{1}{\pi i} \int\left(\frac{D_{t} \zeta(\alpha, t)-D_{t} \zeta(\beta, t)}{\zeta(\alpha, t)-\zeta(\beta, t)}\right)^{2} \partial_{\beta} \overline{D_{t} \zeta}(\beta, t) d \beta\right\}\right)
\end{aligned}
$$

and

$$
\begin{aligned}
\mathcal{H} f(\alpha, t) & =U_{k}^{-1} \mathfrak{H} U_{k} f(\alpha, t)=\frac{1}{\pi i} p . v . \int \frac{f(\beta, t) \zeta_{\beta}(\beta, t)}{\zeta(\alpha, t)-\zeta(\beta, t)} d \beta, \\
\mathcal{K}^{*} f(\alpha, t) & =p . v . \int \operatorname{Re}\left\{\frac{-1}{\pi i} \frac{\zeta_{\alpha}}{\left|\zeta_{\alpha}\right|} \frac{\left|\zeta_{\beta}(\beta, t)\right|}{(\zeta(\alpha, t)-\zeta(\beta, t))}\right\} f(\beta, t) d \beta .
\end{aligned}
$$

Notice the remarkable similarities between equations (2.6)-(2.5) and (2.11)(2.12). In particular, the structures of the terms in (2.6)-(2.5) do not change under the change of variables. This makes it convenient for us to work in another coordinate system and to choose a different coordinate system when there is advantage to do so. In fact, using (2.11)-(2.12) (and the corresponding 3D version) one can prove the local in time well-posedness of the water wave problem in this arbitrarily chosen coordinate system using the same analysis as in $[\mathbf{3 2}, \mathbf{3 3}]$.

We now turn to the question of long time behavior of the solutions for the water wave equation (1.1) for small initial data.

\section{Global and almost global well-posedness of the $2 \mathrm{D}$ and $3 \mathrm{D}$ water wave problem}

Our study of the global in time behavior of the water wave equation (1.1) further uses two new ideas, one is the dispersive aspect of the water wave motion, another is a better understanding of the nature of the nonlinearity of (1.1).

Let $\mathfrak{u}=\xi_{t}$. Linearizing the quasi-linear system (2.6)-(2.5) (or the quasilinear system (5.21)-(5.22) of [33] for 3D water waves) at the zero solution, we obtain

$$
\partial_{t}^{2} \mathfrak{u}+|D| \mathfrak{u}=F\left(\mathfrak{u}_{t},|D| \mathfrak{u}\right), \quad(\alpha, t) \in \mathbb{R}^{n-1} \times \mathbb{R}
$$

where $|D|=\sqrt{-\Delta}, \Delta$ is the Laplacian in $\mathbb{R}^{n-1}$ for n-D water waves, $F$ consists of the nonlinear terms. It is not surprising that $F$ contains quadratic 
nonlinear terms. We know the dispersion relation of the free equation

$$
\partial_{t}^{2} \mathfrak{u}+|D| \mathfrak{u}=0
$$

is

$$
\omega^{2}=|k|
$$

for plane wave solutions $\mathfrak{u}=e^{i(k \cdot \alpha+\omega t)}$. So waves of wave number $k$ travel with phase velocity $\frac{\omega}{|k|} \hat{k}$, where $\hat{k}=\frac{k}{|k|}$, equation (3.1) is dispersive. We know for a large class $\mathcal{B}$ of smooth initial data, the solution of the linear equation (3.2) exists for all time and remains smooth, and its $L^{\infty}$ norm decays with rate $1 / t^{\frac{n-1}{2}}$. But nonlinear interactions can cause blow-up of the solutions at finite time. So to answer the question that for small data in $\mathcal{B}$, for how long does the solution of the nonlinear equation (3.1) remain smooth, we need to know for how long does the linear part of the equation (3.1) remain dominant. The weaker the nonlinear interaction, the longer the solution remains smooth. For small data, quadratic interactions are in general stronger than the cubic and higher order interactions.

To illustrate this idea, we look at the following model equation with the $(p+1)$ th-order nonlinearity:

$$
\partial_{t}^{2} \mathfrak{u}+|D| \mathfrak{u}=\left(\partial_{t} \mathfrak{u}\right)^{p+1}, \quad(\alpha, t) \in \mathbb{R}^{n-1} \times \mathbb{R}
$$

Suppose we can prove such decay estimates for the solution: for $i \leq s-10$,

$$
\left|\partial^{i} \partial_{t} \mathfrak{u}(t)\right|_{L^{\infty}} \lesssim(1+t)^{-\frac{n-1}{2}} E_{s}(t)^{1 / 2}
$$

where $\partial$ is some kind of derivatives,

$$
E_{s}(t)=\sum_{|j| \leq s} \int\left|\partial^{j} \partial_{t} \mathfrak{u}(\alpha, t)\right|^{2}+\left.\left.\left|\partial^{j}\right| D\right|^{1 / 2} \mathfrak{u}(\alpha, t)\right|^{2} d \alpha
$$

Then we can derive energy estimates for $s \geq 20$,

$$
\frac{d}{d t} E_{s}(t) \lesssim(1+t)^{-\frac{(n-1) p}{2}} E_{s}(t)^{p / 2+1}
$$

therefore

$$
E_{s}(0)^{-p / 2}-E_{s}(T)^{-p / 2} \lesssim \int_{0}^{T}(1+t)^{-\frac{(n-1) p}{2}} d t
$$

Heuristically, we expect to prove existence of solutions of (3.4) for as long as the energy $E_{s}(t)$ remains finite. If $p=1$, i.e. if the nonlinear term in (3.4) is quadratic, then for both $n=2$ and $n=3$, the integral $\int_{0}^{\infty}(1+t)^{-\frac{(n-1) p}{2}} d t=$ $\infty$, and we would not be able to conclude solutions exist for all time (for small initial data) from this analysis. In fact, if $p=1$ and $n=2$, the existence time we expect from this analysis would be of order $O\left(\epsilon^{-2}\right)$ for data of size $\epsilon$. On the other hand, if $p \geq 2$, i.e. if there is no quadratic nonlinearity, then we can expect to prove longer time existence for solutions of (3.4) starting from small initial data. In fact, we can expect for $n=2, p=2$, an existence 
time period of $\left[0, e^{c / \epsilon^{2}}\right]$; and for $n=3, p=2$, an existence time period $[0, \infty)$ for data of size $\epsilon$, when $\epsilon$ is sufficiently small.

So the question now is whether there is another unknown $\mathfrak{v}$ that satisfies an equation of the type

$$
\partial_{t}^{2} \mathfrak{v}+|D| \mathfrak{v}=F_{1}\left(\mathfrak{v}_{t},|D| \mathfrak{v}, \mathfrak{u}_{t},|D| \mathfrak{u}\right)
$$

with $F_{1}$ containing no quadratic nonlinearities and $\|\mathfrak{v}\| \approx\|\mathfrak{u}\|$ in various norms $\|\cdot\|$ involved in the course of analysis. The idea of finding such an unknown $\mathfrak{v}$ is the so called method of normal forms, originally introduced by Poincaré in solving ordinary differential equations. Certainly in most cases, one should not expect such a new unknown $\mathfrak{v}$ exist. For quadratic Klein-Gordon equation however, Shatah $[\mathbf{2 4}]$ succeeded in finding a bilinear normal form transformation of the type

$$
v=u+B(u, u)
$$

with $B(u, u)$ bilinear, canceling out the quadratic nonlinear terms in the Klein-Gordon equation, and satisfying the norm equivalence $\|u+B(u, u)\| \approx$ $\|u\|$ for small $\|u\|$. However using the bilinear normal form ansatz $\mathfrak{v}=$ $\mathfrak{u}+\mathfrak{B}(\mathfrak{u}, \mathfrak{u})$, we found that in order to cancel the quadratic terms in the water wave system (2.6)-(2.5) (or (5.21)-(5.22) of [33] for 3D water waves), the bilinear form $\mathfrak{B}(\mathfrak{u}, \mathfrak{u})$ must contain a small divisor of the type

$$
\mathfrak{B}(\mathfrak{u}, \mathfrak{u})=\iint e^{i \alpha \cdot\left(\eta_{1}+\eta_{2}\right)} m\left(\eta_{1}, \eta_{2}\right) \hat{\mathfrak{u}}\left(\eta_{1}\right) \hat{\mathfrak{u}}\left(\eta_{2}\right) d \eta_{1} d \eta_{2}
$$

where

$$
\varlimsup_{\eta_{1} \rightarrow 0}\left|\eta_{1}\right| m\left(\eta_{1}, \eta_{2}\right) \neq 0
$$

Proving $\|\mathfrak{u}+\mathfrak{B}(\mathfrak{u}, \mathfrak{u})\| \approx\|\mathfrak{u}\|$ for small $\|\mathfrak{u}\|$ in such norms $\|\cdot\|=\|\cdot\|_{L^{2}\left(\mathbb{R}^{n-1}\right)}$ is then impossible, as it amounts to proving such an inequality $\|f\|_{L^{2}\left(\mathbb{R}^{n-1}\right)} \lesssim$ $\|\nabla f\|_{L^{2}\left(\mathbb{R}^{n-1}\right)}$.

After further analysis and reformulation of the water wave system (2.6)(2.5) (or (5.21)-(5.22) of [33] for 3d water waves), we realized that the small divisor might be attributable to the particular coordinate system used in (2.6)-(2.5) (or (5.21)-(5.22) of [33] for 3 d water waves), choosing an appropriate coordinate system (if it exists) might resolve this difficulty. Indeed through much further effort, we find new unknowns and new coordinate systems for both the two and three dimensional water waves, such that in these coordinate systems, the new unknown functions satisfy equations with no quadratic nonlinearities.

3.1. The transformation for the $2 \mathrm{D}$ water waves. We give the transformation we constructed for the $2 \mathrm{D}$ water waves, c.f. [34]. 
Proposition 3.1 (Proposition 2.3 of [34]). Let $\xi=\xi(\alpha, t)$ be a solution of the $2 d$ water wave system $(2.2)$. Let $\Pi=(I-\mathfrak{H})(\xi-\bar{\xi}), k=k(\cdot, t): \mathbb{R} \rightarrow \mathbb{R}$ be a diffeomorphism. Let $\zeta=\xi \circ k^{-1}, D_{t} \zeta=\xi_{t} \circ k^{-1}$. We have

$$
\begin{aligned}
\left(D_{t}^{2}\right. & \left.-i A \partial_{\alpha}\right)\left(\Pi \circ k^{-1}\right) \\
= & \frac{4}{\pi} \int \frac{\left(D_{t} \zeta(\alpha, t)-D_{t} \zeta(\beta, t)\right)(\operatorname{Im} \zeta(\alpha, t)-\operatorname{Im} \zeta(\beta, t))}{|\zeta(\alpha, t)-\zeta(\beta, t)|^{2}} \partial_{\beta} D_{t} \zeta(\beta, t) d \beta \\
& +\frac{2}{\pi} \int\left(\frac{D_{t} \zeta(\alpha, t)-D_{t} \zeta(\beta, t)}{\zeta(\alpha, t)-\zeta(\beta, t)}\right)^{2} \partial_{\beta} \operatorname{Im} \zeta(\beta, t) d \beta
\end{aligned}
$$

Here $\operatorname{Im} \zeta$ is the imaginary part of $\zeta$.

Notice that the right hand side of equation (3.8) is cubicly small if the velocity $D_{t} \zeta$, the height function $\operatorname{Im} \zeta$ of the interface (and their derivatives) are small. But the left hand side of (3.8) still contains quadratic nonlinearities. We resolved this difficulty by choosing an appropriate coordinate change $k$.

Let $^{23}$

$$
k(\alpha, t)=\bar{\xi}(\alpha, t)+\frac{1}{2}(I+\mathfrak{H})(I+\mathfrak{K})^{-1}(\xi-\bar{\xi})
$$

where $\mathfrak{K}=\operatorname{Re} \mathfrak{H}$ is the double layered potential operator. In Proposition 3.2 we will see that for the diffeomorphism $k$ as defined by (3.9), the quantities $b=k_{t} \circ k^{-1}$ and $A-1=\left(\mathfrak{a} k_{\alpha}\right) \circ k^{-1}-1$ are quadratically small provided the velocity $D_{t} \zeta$ and the height function $\operatorname{Im} \zeta$ of the interface (and their derivatives) are small. Therefore when the diffeomorephism $k$ is given by (3.9), the left hand side of (3.8) is a linear term $\left(\partial_{t}^{2}-i \partial_{\alpha}\right)\left(\Pi \circ k^{-1}\right)$ plus some terms that are cubicly small.

Proposition 3.2 (Proposition 2.4 of [34]). Let $k$ be as given in (3.9), $b=k_{t} \circ k^{-1}$ and $A=\left(\mathfrak{a} k_{\alpha}\right) \circ k^{-1}$. We have

$$
\begin{aligned}
(I-\mathcal{H}) b & =-\left[D_{t} \zeta, \mathcal{H}\right] \frac{\bar{\zeta}_{\alpha}-1}{\zeta_{\alpha}} \\
(I-\mathcal{H})(A-1) & =i\left[D_{t} \zeta, \mathcal{H}\right] \frac{\partial_{\alpha} \overline{D_{t} \zeta}}{\zeta_{\alpha}}+i\left[D_{t}^{2} \zeta, \mathcal{H}\right] \frac{\bar{\zeta}_{\alpha}-1}{\zeta_{\alpha}}
\end{aligned}
$$

\footnotetext{
${ }^{2}$ The first coordinate change as defined in (2.18) of [34] used the Riemann mapping. However there is no Riemann mapping in 3D. To construct the coordinate change for the $3 \mathrm{D}$ water waves, we realized that the only property used of the Riemann mapping is that it is a holomorphic function in the fluid domain $\Omega(t)$ with its imaginary part equal to zero on $\Sigma(t)$. We therefore came up with a construction for the 3D water waves using this idea. The current formulation as in (3.9) also uses this idea and is used in the author's recent work with $\mathrm{N}$. Totz on a rigorous justification of the modulation approximation for the $2 \mathrm{D}$ water wave problem $[\mathbf{3 1}]$.

${ }^{3}$ This $k=k(\cdot, t): \mathbb{R} \rightarrow \mathbb{R}$ as defined in (3.9) is a diffeomorphism provided the interface $\xi=\xi(\cdot, t)$ is a small perturbation from the flat wave. This can be proved in a similar way as that in $[\mathbf{3 4}]$.
} 
Therefore the quantity $\Pi \circ k^{-1}=(I-\mathcal{H})(\zeta-\bar{\zeta})$, with the coordinate change $k$ given as in (3.9), satisfies an equation of the type $\left(\partial_{t}^{2}-i \partial_{\alpha}\right)(\Pi \circ$ $\left.k^{-1}\right)=\mathcal{G}$ with $\mathcal{G}$ containing only nonlinear terms of cubic and higher orders. We make the following remarks:

\section{REMARK $3.3 .^{4}$}

1. The transformation $I-\mathfrak{H}$ and the coordinate change $k$ as given in (3.9) are fully nonlinear in terms of the unknown function $\zeta$ and its derivatives.

2. The bilinear part of the quantity $(I-\mathfrak{H})(\xi-\bar{\xi})$ has a bounded Fourier symbol. The coordinate change $k$ has taken care of the difficulty of the small divisor in the Fourier symbol of the bilinear normal form transformation. This can be easily seen in the following calculation.

Assume that a normal form transformation $\mathfrak{G}$ is exactly obtained from the original unknown $\mathfrak{g}$ by a change of coordinates $k^{-1}$, i.e. $\mathfrak{G}(X)=\mathfrak{g}\left(k^{-1}(X)\right)$, with $k(X)-X$ a small quantity depending on $\mathfrak{g}$. By the chain rule we know $\|\mathfrak{G}\| \approx\|\mathfrak{g}\|$ in various norms $\|\cdot\|$ provided the norms of $\nabla k^{-1}-I$ are finite, here $I$ is the identity map. On the other hand, from the Taylor expansion

$$
\mathfrak{G}(X)=\mathfrak{g}\left(k^{-1}(X)\right)=\mathfrak{g}(X)+\nabla \mathfrak{g}(X) \cdot\left(k^{-1}(X)-X\right)+\ldots,
$$

we know that if one only retains the linear and bilinear parts of the transformation $\mathfrak{g} \circ k^{-1}$, then one gets

$$
\mathfrak{G}_{1}(X)=\mathfrak{g}(X)+\nabla \mathfrak{g}(X) \cdot\left(k^{-1}(X)-X\right)^{5}
$$

To obtain $\left\|\mathfrak{G}_{1}\right\| \approx\|\mathfrak{g}\|$ in various norms $\|\cdot\|$ for small $\|\mathfrak{g}\|$, one cannot avoid engaging the smallness of the norms of the primitive $k^{-1}(X)-X$, and the dependence on $\nabla \mathfrak{g}$ in the quadratic term can also be problematic. Therefore if one uses a full nonlinear transformation, in this case a full coordinate change, one doesn't encounter the problem of small divisors as one would if one considered a transformation consisting of only linear and bilinear forms of $\mathfrak{g}$.

3. Notice that the linear operator of the equation (3.8) is $\partial_{t}^{2}-i \partial_{\alpha}$. The difference of $\left(\partial_{t}^{2}+|D|\right)\left(\Pi \circ k^{-1}\right)$ and $\left(\partial_{t}^{2}-i \partial_{\alpha}\right)\left(\Pi \circ k^{-1}\right)$ contains quadratic nonlinearities.

The proofs of Propositions 3.1, 3.2 use the following identities, c.f. [34]. Let $\xi=\xi(\cdot, t)$ define a non-selfintersecting curve, and $\mathfrak{H}$ be the Hilbert transform as defined in (2.1).

\footnotetext{
${ }^{4}$ For more detailed discussion of the normal form transformation of the water wave equations, we refer the reader to Appendix $\mathrm{C}$ of [35].

${ }^{5}$ This suggests an algorithm to handle problems where the bilinear normal form transformation contain small divisors attributable to a change of coordinates.
} 
Lemma 3.4 (Lemma 2.1 of $[\mathbf{3 4}])$. Assume that $\xi_{t}, \xi_{\alpha}-1 \in C^{1}([0, T]$, $\left.H^{1}(\mathbb{R})\right), f \in C^{1}(\mathbb{R} \times(0, T))$ satisfies $f_{\alpha}(\alpha, t) \rightarrow 0$, as $|\alpha| \rightarrow \infty$. We have

$$
\begin{aligned}
{\left[\partial_{t}, \mathfrak{H}\right] f } & =\left[\xi_{t}, \mathfrak{H}\right] \frac{f_{\alpha}}{\xi_{\alpha}} \\
{\left[\partial_{t}^{2}, \mathfrak{H}\right] f } & =\left[\xi_{t t}, \mathfrak{H}\right] \frac{f_{\alpha}}{\xi_{\alpha}}+2\left[\xi_{t}, \mathfrak{H}\right] \frac{f_{t \alpha}}{\xi_{\alpha}}-\frac{1}{\pi i} \int\left(\frac{\xi_{t}(\alpha, t)-\xi_{t}(\beta, t)}{\xi(\alpha, t)-\xi(\beta, t)}\right)^{2} f_{\beta} d \beta \\
{\left[\mathfrak{a} \partial_{\alpha}, \mathfrak{H}\right] f } & =\left[\mathfrak{a} \xi_{\alpha}, \mathfrak{H}\right] \frac{f_{\alpha}}{\xi_{\alpha}}, \quad \partial_{\alpha} \mathfrak{H} f=\xi_{\alpha} \mathfrak{H} \frac{f_{\alpha}}{\xi_{\alpha}} \\
{\left[\partial_{t}^{2}-i \mathfrak{a} \partial_{\alpha}, \mathfrak{H}\right] f } & =2\left[\xi_{t}, \mathfrak{H}\right] \frac{f_{t \alpha}}{\xi_{\alpha}}-\frac{1}{\pi i} \int\left(\frac{\xi_{t}(\alpha, t)-\xi_{t}(\beta, t)}{\xi(\alpha, t)-\xi(\beta, t)}\right)^{2} f_{\beta} d \beta \\
(I-\mathfrak{H})\left(-i \mathfrak{a}_{t} \bar{\xi}_{\alpha}\right) & =2\left[\xi_{t t}, \mathfrak{H}\right] \frac{\bar{\xi}_{t \alpha}}{\xi_{\alpha}}+2\left[\xi_{t}, \mathfrak{H}\right] \frac{\bar{\xi}_{t t \alpha}}{\xi_{\alpha}}-\frac{1}{\pi i} \int\left(\frac{\xi_{t}(\alpha, t)-\xi_{t}(\beta, t)}{\xi(\alpha, t)-\xi(\beta, t)}\right)^{2} \bar{\xi}_{t \beta} d \beta
\end{aligned}
$$

The proof of Lemma 3.4 is straightforwardly integration by parts, we omit the proof.

Let $\Omega$ be a $C^{1}$ domain in the complex plane. Assume that the boundary of $\Omega$ is parametrized by $z=z(\alpha), \alpha \in \mathbb{R}$, oriented in the clockwise sense, and there exist constants $\mu_{1}, \mu_{2}>0$, such that $\mu_{1}|\alpha-\beta| \leq|z(\alpha)-z(\beta)| \leq$ $\mu_{2}|\alpha-\beta|$ for all $\alpha, \beta \in \mathbb{R}$. Let

$$
\mathbf{H} f(\alpha)=\frac{1}{\pi i} p \cdot v \cdot \int \frac{z_{\beta}(\beta)}{z(\alpha)-z(\beta)} f(\beta) d \beta
$$

be the associated Hilbert transform. We know $\mathbf{H}^{2}=I$ in $L^{2}$, we use the convention $\mathbf{H} 1=0$. We have

LEMMA 3.5 (Lemma 2.2 of $[\mathbf{3 4}]$ ).

1. $f(\cdot)=F(z(\cdot)) \in L^{2}(\partial \Omega)$ is the boundary value of a holomorphic function $F$ in $\Omega$ if and only if $f=\mathbf{H} f$.

2. If $f=\mathbf{H} f, g=\mathbf{H} g$, then $[f, \mathbf{H}] g=0$.

3. For any $f, g \in L^{2}(\partial \Omega)$, we have $[f, \mathbf{H}] \mathbf{H} g=-[\mathbf{H} f, \mathbf{H}] g$.

The first statement in Lemma 3.5 is a consequence of the Cauchy integral formula. The second statement follows from the fact that the product of holomorphic functions is holomorphic. For details of the proof, see [34].

We now give the proofs for Propositions 3.1, 3.2. ${ }^{6}$ We first prove (3.8). We have, for $\xi=\xi(\cdot, t)$ satisfying the water wave system $(2.2)$,

$$
\begin{aligned}
\left(\partial_{t}^{2}-i \mathfrak{a} \partial_{\alpha}\right)\{(I-\mathfrak{H})(\xi-\bar{\xi})\}= & (I-\mathfrak{H})\left\{\left(\partial_{t}^{2}-i \mathfrak{a} \partial_{\alpha}\right)(\xi-\bar{\xi})\right\} \\
& -\left[\partial_{t}^{2}-i \mathfrak{a} \partial_{\alpha}, \mathfrak{H}\right](\xi-\bar{\xi})
\end{aligned}
$$

\footnotetext{
${ }^{6}$ The proofs are taken from $[\mathbf{3 4}]$.
} 


$$
\begin{aligned}
= & (I-\mathfrak{H})\left(-2 \bar{\xi}_{t t}\right)-2\left[\xi_{t}, \mathfrak{H}\right] \frac{\xi_{t \alpha}-\bar{\xi}_{t \alpha}}{\xi_{\alpha}} \\
& +\frac{1}{\pi i} \int\left(\frac{\xi_{t}(\alpha, t)-\xi_{t}(\beta, t)}{\xi(\alpha, t)-\xi(\beta, t)}\right)^{2} \partial_{\beta}(\xi(\beta, t)-\bar{\xi}(\beta, t)) d \beta \\
= & -2\left[\xi_{t}, \mathfrak{H}\right] \frac{\xi_{t \alpha}}{\xi_{\alpha}}+\frac{1}{\pi i} \int\left(\frac{\xi_{t}(\alpha, t)-\xi_{t}(\beta, t)}{\xi(\alpha, t)-\xi(\beta, t)}\right)^{2}(\xi-\bar{\xi})_{\beta} d \beta \\
= & -2\left[\xi_{t}, \mathfrak{H} \frac{1}{\xi_{\alpha}}+\overline{\mathfrak{H}} \frac{1}{\bar{\xi}_{\alpha}}\right] \xi_{t \alpha} \\
& +\frac{1}{\pi i} \int\left(\frac{\xi_{t}(\alpha, t)-\xi_{t}(\beta, t)}{\xi(\alpha, t)-\xi(\beta, t)}\right)^{2}\left(\xi_{\beta}-\bar{\xi}_{\beta}\right) d \beta
\end{aligned}
$$

Here in the second and third steps we used (2.2) and Lemma 3.4, and in the fourth step we inserted the term $\left[\xi_{t}, \overline{\mathfrak{H}} \frac{1}{\bar{\xi}_{\alpha}}\right] \xi_{t \alpha}$ to make it an at least cubic nonlinear term. $\left[\xi_{t}, \overline{\mathfrak{H}}_{\bar{\xi}_{\alpha}} \frac{1}{\xi_{t \alpha}}=0\right.$ because both $\bar{\xi}_{t}$ and $\frac{\bar{\xi}_{t \alpha}}{\xi_{\alpha}}$ are traces of some holomorphic functions in $\Omega(t)$ and because of the part 2 of Lemma 3.5. Applying the change of coordinates $U_{k^{-1}}$ to (3.14), and expanding the first term in the last equality, we arrive at (3.8).

Now for the proof of Proposition 3.2, we notice that for $k$ be as given in $(3.9)$,

$$
k(\alpha, t)-\bar{\xi}(\alpha, t)=\frac{1}{2}(I+\mathfrak{H})(I+\mathfrak{K})^{-1}(\xi-\bar{\xi})
$$

is the trace on $\Sigma(t)$ of a holomorphic function in $\Omega(t)$, so (3.10) can be proved in exactly the same way as that of Proposition 2.4 in [34]: Let $\Phi$ be the holomorphic function in $\Omega(t)$, such that

$$
\bar{\xi}(\alpha, t)-k(\alpha, t)=\Phi(\xi(\alpha, t), t) .
$$

We have,

$$
\bar{\xi}_{t}-k_{t}=\Phi_{t}+\Phi_{\xi} \xi_{t}, \quad \bar{\xi}_{\alpha}-k_{\alpha}=\Phi_{\xi} \xi_{\alpha} .
$$

Using the fact $\Phi_{t}, \Phi_{\xi}$ are holomorphic in $\Omega(t)$ and Lemma 3.5, we obtain

$$
\begin{aligned}
-(I-\mathfrak{H}) k_{t} & =(I-\mathfrak{H})\left(\bar{\xi}_{t}-k_{t}\right) \\
& =(I-\mathfrak{H})\left\{\Phi_{\xi} \xi_{t}\right\}=\left[\xi_{t}, \mathfrak{H}\right] \Phi_{\xi}=\left[\xi_{t}, \mathfrak{H}\right] \frac{\bar{\xi}_{\alpha}-k_{\alpha}}{\xi_{\alpha}} .
\end{aligned}
$$

Now using (3.15), (2.2), and the fact that $\Phi_{\xi}$ is holomorphic in $\Omega(t)$, we also have

$$
(I-\mathfrak{H})\left(i \mathfrak{a} \bar{\xi}_{\alpha}-i \mathfrak{a} k_{\alpha}\right)=(I-\mathfrak{H})\left(i \mathfrak{a} \xi_{\alpha} \Phi_{\xi}\right)=\left[\xi_{t t}, \mathfrak{H}\right] \Phi_{\xi}
$$

therefore using Lemma 3.4, (2.2) and (3.15),

$$
\begin{aligned}
-(I-\mathfrak{H})\left(i \mathfrak{a} k_{\alpha}\right) & =-(I-\mathfrak{H})\left(i \mathfrak{a} \bar{\xi}_{\alpha}\right)+\left[\xi_{t t}, \mathfrak{H}\right] \Phi_{\xi} \\
& =-i+\left[\xi_{t}, \mathfrak{H}\right] \frac{\bar{\xi}_{t \alpha}}{\xi_{\alpha}}+\left[\xi_{t t}, \mathfrak{H}\right] \frac{\bar{\xi}_{\alpha}-k_{\alpha}}{\xi_{\alpha}}
\end{aligned}
$$

Applying the change of variable $U_{k}^{-1}$ to $(3.16),(3.17)$, we arrive at (3.10). 
3.2. The transformation for the $3 \mathrm{D}$ water waves. We study the global in time behavior of the $3 \mathrm{D}$ water wave problem in the setting of the Clifford Algebra $\mathcal{C}\left(V_{2}\right)$. We further introduce the following notations.

We know an element $\sigma \in \mathcal{C}\left(V_{2}\right)$ has a unique representation $\sigma=\sigma_{0}+$ $\sum_{i=1}^{3} \sigma_{i} e_{i}$, with $\sigma_{i} \in \mathbb{R}$ for $0 \leq i \leq 3$; We call $\sigma$ a vector if $\sigma_{0}=0$. If not specified, we always assume in such an expression $\sigma=\sigma_{0}+\sum_{i=1}^{3} \sigma_{i} e_{i}$ that $\sigma_{i} \in \mathbb{R}$ for $0 \leq i \leq 3$. We define $\bar{\sigma}=e_{3} \sigma e_{3}$, the conjugate of $\sigma$. We identify a point or a vector $\xi=(x, y, z) \in \mathbb{R}^{3}$ with its $\mathcal{C}\left(V_{2}\right)$ counterpart $\xi=x e_{1}+y e_{2}+z e_{3}$. For vectors $\xi, \eta \in \mathcal{C}\left(V_{2}\right)$, we know

$$
\xi \eta=-\xi \cdot \eta+\xi \times \eta
$$

where $\xi \cdot \eta$ is the dot product, $\xi \times \eta$ the cross product. For vectors $\xi, \zeta, \eta$, $\xi(\zeta \times \eta)$ is obtained by first finding the cross product $\zeta \times \eta$, then regard it as a Clifford vector and calculating its multiplication with $\xi$ by the rule (2.7). We write $\nabla=\left(\partial_{x}, \partial_{y}, \partial_{z}\right)$. We abbreviate notations such as

$$
\begin{aligned}
\mathfrak{H}_{\Sigma} f(\alpha, \beta) & =\iint K\left(\eta\left(\alpha^{\prime}, \beta^{\prime}\right)-\eta(\alpha, \beta)\right)\left(\eta_{\alpha^{\prime}}^{\prime} \times \eta_{\beta^{\prime}}^{\prime}\right) f\left(\alpha^{\prime}, \beta^{\prime}\right) d \alpha^{\prime} d \beta^{\prime} \\
& =\iint K\left(\eta^{\prime}-\eta\right)\left(\eta_{\alpha^{\prime}}^{\prime} \times \eta_{\beta^{\prime}}^{\prime}\right) f^{\prime} d \alpha^{\prime} d \beta^{\prime}=\iint K N^{\prime} f^{\prime} d \alpha^{\prime} d \beta^{\prime} .
\end{aligned}
$$

Similar to the $2 \mathrm{D}$ case, we also have $\mathfrak{H}_{\Sigma}^{2}=I$ in $L^{2}$; we use the convention $\mathfrak{H}_{\Sigma} 1=0$.

We now give the transformation for the $3 \mathrm{D}$ water wave equation. Let $\Omega(t)$ be the fluid region, $\Sigma(t)$ be the fluid interface in $\mathbb{R}^{3}$. Let $\Sigma(t)$ : $\xi(\alpha, \beta, t)=x(\alpha, \beta, t) e_{1}+y(\alpha, \beta, t) e_{2}+z(\alpha, \beta, t) e_{3},(\alpha, \beta) \in \mathbb{R}^{2}$ be the parameterization of the interface $\Sigma(t)$ at time $t$ in Lagrangian coordinates $(\alpha, \beta)$ with $N=\xi_{\alpha} \times \xi_{\beta}$ pointing out of the fluid domain $\Omega(t)$. Let $\mathfrak{H}=\mathfrak{H}_{\Sigma(t)}$ be defined as in (2.8), and

$$
\mathfrak{a}=-\frac{1}{|N|} \frac{\partial P}{\partial \mathbf{n}} .
$$

We know from $[\mathbf{3 3}]$ that $\mathfrak{a}>0$ and equation $(1.1)(n=3)$ is equivalent to the following nonlinear system defined on the interface $\Sigma(t)$ :

$$
\left\{\begin{array}{l}
\xi_{t t}+e_{3}=\mathfrak{a} N \\
\xi_{t}=\mathfrak{H} \xi_{t}
\end{array}\right.
$$

Let $U_{g} f(\alpha, \beta, t)=f(g(\alpha, \beta, t), t)=f \circ g(\alpha, \beta, t)$. For fixed $t$, let $k=$ $k(\cdot, t)=k_{1} e_{1}+k_{2} e_{2}: \mathbb{R}^{2} \rightarrow \mathbb{R}^{2}$ be a diffeomorphism with its Jacobian $J(k(t))>0$. Let $k^{-1}$ be such that $k \circ k^{-1}(\alpha, \beta, t)=\alpha e_{1}+\beta e_{2}$. Define

$$
\zeta=\xi \circ k^{-1}, \quad b=k_{t} \circ k^{-1}, \quad A \circ k e_{3}=\mathfrak{a} J(k) e_{3}=\mathfrak{a} k_{\alpha} \times k_{\beta}
$$


Let $D_{t}=U_{k}^{-1} \partial_{t} U_{k}$ be the material derivative, $\mathcal{N}=\zeta_{\alpha} \times \zeta_{\beta}$. By a simple application of the chain rule, we know

$$
D_{t}=\partial_{t}+b \cdot\left(\partial_{\alpha}, \partial_{\beta}\right), \quad U_{k}^{-1}(\mathfrak{a} N \times \nabla) U_{k}=A \mathcal{N} \times \nabla=A\left(\zeta_{\beta} \partial_{\alpha}-\zeta_{\alpha} \partial_{\beta}\right),
$$

and $U_{k}^{-1} \mathfrak{H} U_{k}=\mathcal{H}$, with

$$
\mathcal{H} f(\alpha, \beta, t)=\iint K\left(\zeta\left(\alpha^{\prime}, \beta^{\prime}, t\right)-\zeta(\alpha, \beta, t)\right)\left(\zeta_{\alpha^{\prime}}^{\prime} \times \zeta_{\beta^{\prime}}^{\prime}\right) f\left(\alpha^{\prime}, \beta^{\prime}, t\right) d \alpha^{\prime} d \beta^{\prime}
$$

We have

Proposition 3.6 (Proposition 1.3 of $[\mathbf{3 5}]$ ). Let $\xi=\xi(\alpha, \beta, t)$ be a solution of the $3 d$ water wave system (3.19). Let $\Pi=(I-\mathfrak{H})(\xi-\bar{\xi})$, and for fixed $t, k(\cdot, t): \mathbb{R}^{2} \rightarrow \mathbb{R}^{2}$ be a diffeomorphism. We have

$$
\begin{aligned}
\left(D_{t}^{2}-\right. & A \mathcal{N} \times \nabla)\left(\Pi \circ k^{-1}\right) \\
= & 2 \iint K\left(\zeta^{\prime}-\zeta\right)\left(D_{t} \zeta-D_{t}^{\prime} \zeta^{\prime}\right) \times\left(\zeta_{\beta^{\prime}}^{\prime} \partial_{\alpha^{\prime}}-\zeta_{\alpha^{\prime}}^{\prime} \partial_{\beta^{\prime}}\right) \overline{D_{t}^{\prime} \zeta^{\prime}} d \alpha^{\prime} d \beta^{\prime} \\
& -\iint K\left(\zeta^{\prime}-\zeta\right)\left(D_{t} \zeta-D_{t}^{\prime} \zeta^{\prime}\right) \times\left(\left(D_{t}^{\prime} \zeta^{\prime}\right)_{\beta^{\prime}} \partial_{\alpha^{\prime}}\right. \\
& \left.-\left(D_{t}^{\prime} \zeta^{\prime}\right)_{\alpha^{\prime}} \partial_{\beta^{\prime}}\right)\left(\zeta^{\prime}-\overline{\zeta^{\prime}}\right) d \alpha^{\prime} d \beta^{\prime} \\
& -\iint D_{t} K\left(\zeta^{\prime}-\zeta\right)\left(D_{t} \zeta-D_{t}^{\prime} \zeta^{\prime}\right) \times\left(\zeta_{\beta^{\prime}}^{\prime} \partial_{\alpha^{\prime}}-\zeta_{\alpha^{\prime}}^{\prime} \partial_{\beta^{\prime}}\right)\left(\zeta^{\prime}-\bar{\zeta}^{\prime}\right) d \alpha^{\prime} d \beta^{\prime}
\end{aligned}
$$

We see that the second and third terms in the right hand side of (3.23) are cubicly small provided the velocity $D_{t} \zeta$ and the steepness of the height function $\partial_{\alpha}(\zeta-\bar{\zeta}), \partial_{\beta}(\zeta-\bar{\zeta})$ are small, while the first term appears to be only quadratically small. Unlike the $2 \mathrm{D}$ case, multiplications of Clifford analytic functions are not necessarily analytic, so we cannot reduce the first term at the right hand side of equation (3.23) into a cubic form. However we notice that $\overline{D_{t} \zeta}$ is almost analytic ${ }^{7}$ in the air region $\Omega(t)^{c}$, and this implies that the first term is almost analytic in the fluid domain $\Omega(t)$. Notice that the left hand side of (3.23) is almost analytic in the air region. The orthogonality of the projections $(I-\mathcal{H})$ and $(I+\mathcal{H})$ allows us to reduce the first term to cubic in energy estimates.

Now the left hand side of (3.23) still contains quadratic terms. Similar to the $2 \mathrm{D}$ case, this difficulty is resolved by choosing an appropriate coordinate change $k$. Let

$$
k=k(\alpha, \beta, t)=\xi(\alpha, \beta, t)-(I+\mathfrak{H}) z(\alpha, \beta, t) e_{3}+\mathfrak{K} z(\alpha, \beta, t) e_{3}
$$

${ }^{7}$ Since the order of smallness is what matters, here a quantity $X$ of size $O\left(\epsilon^{N}\right)$, in other words of order $N$, is said to be almost analytic in the fluid region $\Omega(t)$ (respectively in the air region $\left.\Omega(t)^{c}\right)$, if $(I-\mathcal{H}) X$ (respectively $(I+\mathcal{H}) X$ ) is at most of size $O\left(\epsilon^{N+1}\right)$, or in other words at least of order $N+1$. 
Here $\mathfrak{K}=\operatorname{Re} \mathfrak{H}$ :

$$
\mathfrak{K} f(\alpha, \beta, t)=-\iint K\left(\xi\left(\alpha^{\prime}, \beta^{\prime}, t\right)-\xi(\alpha, \beta, t)\right) \cdot N^{\prime} f\left(\alpha^{\prime}, \beta^{\prime}, t\right) d \alpha^{\prime} d \beta^{\prime}
$$

is the double layered potential operator. It is clear that the $e_{3}$ component of $k$ as defined in (3.24) is zero. In fact, the real part of $k$ is also zero. This is because

$$
\begin{aligned}
& \iint K\left(\xi^{\prime}-\xi\right) \times\left(\xi_{\alpha^{\prime}}^{\prime} \times \xi_{\beta^{\prime}}^{\prime}\right) z^{\prime} e_{3} d \alpha^{\prime} d \beta^{\prime} \\
& \quad=\iint\left(\xi_{\alpha^{\prime}}^{\prime} \xi_{\beta^{\prime}}^{\prime} \cdot K-\xi_{\beta^{\prime}}^{\prime} \xi_{\alpha^{\prime}}^{\prime} \cdot K\right) z^{\prime} e_{3} d \alpha^{\prime} d \beta^{\prime} \\
& \quad=-2 \iint\left(\xi_{\alpha^{\prime}}^{\prime} \partial_{\beta^{\prime}} \Gamma\left(\xi^{\prime}-\xi\right)-\xi_{\beta^{\prime}}^{\prime} \partial_{\alpha^{\prime}} \Gamma\left(\xi^{\prime}-\xi\right)\right) z^{\prime} e_{3} d \alpha^{\prime} d \beta^{\prime} \\
& \quad=2 \iint \Gamma\left(\xi^{\prime}-\xi\right)\left(\xi_{\alpha^{\prime}}^{\prime} z_{\beta^{\prime}}-\xi_{\beta^{\prime}}^{\prime} z_{\alpha^{\prime}}\right) e_{3} d \alpha^{\prime} d \beta^{\prime} \\
& \quad=2 \iint \Gamma\left(\xi^{\prime}-\xi\right)\left(N_{1}^{\prime} e_{1}+N_{2}^{\prime} e_{2}\right) d \alpha^{\prime} d \beta^{\prime}
\end{aligned}
$$

So

$$
\mathfrak{H} z e_{3}=\mathfrak{K} z e_{3}+2 \iint \Gamma\left(\xi^{\prime}-\xi\right)\left(N_{1}^{\prime} e_{1}+N_{2}^{\prime} e_{2}\right) d \alpha^{\prime} d \beta^{\prime}
$$

This shows that the mapping $k$ defined in (3.24) has only the $e_{1}$ and $e_{2}$ components $k=\left(k_{1}, k_{2}\right)=k_{1} e_{1}+k_{2} e_{2}$. If $\Sigma(t)$ is a graph with small steepness, i.e. if $z_{\alpha}$ and $z_{\beta}$ are small, then the Jacobian of $k=k(\cdot, t)$ : $J(k)=J(k(t))=\partial_{\alpha} k_{1} \partial_{\beta} k_{2}-\partial_{\alpha} k_{2} \partial_{\beta} k_{1}>0$ and $k(\cdot, t): \mathbb{R}^{2} \rightarrow \mathbb{R}^{2}$ defines a valid coordinate change (c.f. [35]).

From the following proposition we see that if $k$ is as given in (3.24), then $b, A-1$ are consisting of only quadratic and higher order terms. Let

$$
\mathcal{K}=\operatorname{Re} \mathcal{H}=U_{k}^{-1} \mathfrak{K} U_{k}, \quad P=\alpha e_{1}+\beta e_{2}, \quad \text { and } \quad \zeta=P+\lambda .
$$

Proposition 3.7 (Proposition 1.4 of [35]). Let $k$ be as given in (3.24). Let $b=k_{t} \circ k^{-1}$ and $A \circ k=\mathfrak{a} J(k)$. We have

$$
\begin{aligned}
b= & \frac{1}{2}(\mathcal{H}-\overline{\mathcal{H}}) \overline{D_{t} \zeta}-\frac{1}{2}\left[D_{t}, \mathcal{H}-\mathcal{K}\right](\zeta-\bar{\zeta})+\frac{1}{2} \mathcal{K}\left(D_{t} \zeta-\overline{D_{t} \zeta}\right) \\
(A-1) e_{3}= & \frac{1}{2}(-\mathcal{H}+\overline{\mathcal{H}}) \overline{D_{t}^{2} \zeta}+\frac{1}{2}\left(\left[D_{t}, \mathcal{H}\right] D_{t} \zeta-\overline{\left[D_{t}, \mathcal{H}\right] D_{t} \zeta}\right) \\
& +\frac{1}{2}[A \mathcal{N} \times \nabla, \mathcal{H}](\zeta-\bar{\zeta})-\frac{1}{2} A \zeta_{\beta} \times\left(\partial_{\alpha} \mathcal{K}(\zeta-\bar{\zeta})\right) \\
& +\frac{1}{2} A \zeta_{\alpha} \times\left(\partial_{\beta} \mathcal{K}(\zeta-\bar{\zeta})\right)+A \partial_{\alpha} \lambda \times \partial_{\beta} \lambda
\end{aligned}
$$

Here $\overline{\mathcal{H}}=e_{3} \mathcal{H} e_{3}$. 
Let $\chi=\Pi \circ k^{-1}$ with $k$ be as defined by (3.24). Therefore the left hand side of equation (3.23) is

$$
\left(\partial_{t}^{2}-e_{2} \partial_{\alpha}+e_{1} \partial_{\beta}\right) \chi-\partial_{\beta} \lambda \partial_{\alpha} \chi+\partial_{\alpha} \lambda \partial_{\beta} \chi+\text { cubic and higher order terms }
$$

The quadratic term $\partial_{\beta} \lambda \partial_{\alpha} \chi-\partial_{\alpha} \lambda \partial_{\beta} \chi$ is new in $3 \mathrm{D}$. We notice that this is one of the null forms studied in [17] and we find that it is also null for our equation and can be written as the factor $1 / t$ times a quadratic expression involving some "invariant vector fields" for $\partial_{t}^{2}-e_{2} \partial_{\alpha}+e_{1} \partial_{\beta}$. Therefore this term is cubic in nature and equation (3.23) is of the type "linear + cubic and higher order perturbations".

3.3. Global in time behavior of solutions for the $2 \mathrm{D}$ and $3 \mathrm{D}$ water waves. With equations (3.8) and (3.23) available now we can study the global in time behavior of small solutions of the $2 \mathrm{D}$ and $3 \mathrm{D}$ water wave equations. We point out that it is more natural to treat $D_{t}^{2}-i A \partial_{\alpha}$ and $D_{t}^{2}-$ $A \mathcal{N} \times \nabla$ as the main operators for the $2 \mathrm{D}$ and $3 \mathrm{D}$ water wave equations than treating them as perturbations of the linear operators $\partial_{t}^{2}-i \partial_{\alpha}$ and $\partial_{t}^{2}-e_{2} \partial_{\alpha}+$ $e_{1} \partial_{\beta}$. The basic idea is what we illustrated with the model equation (3.4); we make this idea rigorous by the method of invariant vector fields. This involves constructing invariant vector fields for the operator $\partial_{t}^{2}-e_{2} \partial_{\alpha}+e_{1} \partial_{\beta}$ (the invariant vector fields for $\partial_{t}^{2}-i \partial_{\alpha}$ for the $2 \mathrm{D}$ water wave are available due to the well studied Schrödinger operator $i \partial_{t}-\partial_{x}^{2}$ ); proving generalized Sobolev inequalities that give $L^{2} \rightarrow L^{\infty}$ estimates with the decay rate $1 / t^{1 / 2}$ for the $2 \mathrm{D}$ and $1 / t$ for the $3 \mathrm{D}$ water waves respectively; using equations (3.8) and (3.23) to show that properly constructed energies that involve invariant vector fields remain bounded for the time period $\left[0, e^{c / \epsilon}\right]$ for the $2 \mathrm{D}$ and for all time for the 3D water waves for data of size $O(\epsilon)$. We point out that not only does the projection $(I-\mathfrak{H})$ give us the quantity $(I-\mathfrak{H})(\xi-\bar{\xi})$, but it is also used in various ways to project away "quadratic noises" in the course of deriving the energy estimates. The almost global well-posedness for the $2 \mathrm{D}$ and global well-posedness for the 3D water wave equations follow from the local well-posedness results, the uniform boundedness of the energies and continuity arguments. We state our main theorems.

Let $|D|=\sqrt{-\Delta}, H^{s}\left(\mathbb{R}^{n-1}\right)=\left\{f \mid(I+|D|)^{s} f \in L^{2}\left(\mathbb{R}^{n-1}\right)\right\}$, with $\|f\|_{H^{s}\left(\mathbb{R}^{n-1}\right)}=\left\|(I+|D|)^{s} f\right\|_{L^{2}\left(\mathbb{R}^{n-1}\right)}$.

2D water waves. Let $s \geq 12, \max \left\{\left[\frac{s}{2}\right]+3,11\right\} \leq l \leq s-1$. Assume initially that

$$
\begin{aligned}
& \xi(\alpha, 0)=(\alpha, y(\alpha)), \quad \xi_{t}(\alpha, 0)=\mathfrak{u}(\alpha), \quad \xi_{t t}(\alpha, 0)=\mathfrak{w}(\alpha) \quad \alpha \in \mathbb{R}, \\
& \mathbf{v}(z, 0)=\mathbf{g}(z), \quad z \in \Omega(0)
\end{aligned}
$$

and the data in (3.29) satisfies the $2 \mathrm{D}$ water wave system (2.2). In particular $\overline{\mathbf{g}}$ is a holomorphic function in the initial fluid domain $\Omega(0)$ and 
$\mathbf{g}(\xi(\alpha, 0))=\mathfrak{u}(\alpha)$. Let $\Gamma=\partial_{\alpha}, \alpha \partial_{\alpha}$. Assume that

$$
\sum_{|j| \leq s-1}\left(\left\|\Gamma^{j} y_{\alpha}\right\|_{H^{1 / 2}(\mathbb{R})}+\left\|\Gamma^{j} \mathfrak{u}\right\|_{H^{3 / 2}(\mathbb{R})}+\left\|\Gamma^{j} \mathfrak{w}\right\|_{H^{1}(\mathbb{R})}\right)<\infty .
$$

Let

$$
\epsilon=\sum_{|j| \leq l}\left(\left\|\Gamma^{j} y\right\|_{H^{1}(\mathbb{R})}+\left\|\Gamma^{j} \mathfrak{u}\right\|_{H^{1}(\mathbb{R})}\right)+\sum_{j \leq l-2}\left\|\left(z \partial_{z}\right)^{j} \overline{\mathbf{g}}\right\|_{L^{2}(\Omega(0))} .
$$

Theorem 3.8 (2D Theorem [34]). There exist $\epsilon_{0}$ and $c>0$, such that for $\epsilon<\epsilon_{0}$, the initial value problem (2.2)-(3.29) has a unique classical solution for the time period $\left[0, e^{c / \epsilon}\right]$. During this time period, the interface is a graph, the solution is as regular as the initial data and remains small. Moreover the $L^{\infty}$ norm of the steepness of the interface $\partial_{\alpha}(\xi-\bar{\xi})$, the velocity $\xi_{t}$ and acceleration $\xi_{t t}$ on the interface decay at rate $1 / t^{1 / 2}$.

3D water waves. Let $s \geq 27, \max \left\{\left[\frac{s}{2}\right]+1,17\right\} \leq l \leq s-10$. Assume that initially

$$
\begin{aligned}
& \xi(\alpha, \beta, 0)=\left(\alpha, \beta, z^{0}(\alpha, \beta)\right), \quad \xi_{t}(\alpha, \beta, 0)=\mathfrak{u}^{0}(\alpha, \beta), \\
& \xi_{t t}(\alpha, \beta, 0)=\mathfrak{w}^{0}(\alpha, \beta),
\end{aligned}
$$

and the data in (3.30) satisfies the $3 \mathrm{D}$ water wave system (3.19). Let $\Gamma=$ $\partial_{\alpha}, \partial_{\beta}, \alpha \partial_{\alpha}+\beta \partial_{\beta}, \alpha \partial_{\beta}-\beta \partial_{\alpha}$. Assume that

$$
\begin{aligned}
& \sum_{\substack{|j| \leq s-1 \\
\partial=\partial_{\alpha}, \partial_{\beta}}}\left\|\Gamma^{j}|D|^{1 / 2} z^{0}\right\|_{L^{2}\left(\mathbb{R}^{2}\right)}+\left\|\Gamma^{j} \partial z^{0}\right\|_{H^{1 / 2}\left(\mathbb{R}^{2}\right)} \\
& \quad+\left\|\Gamma^{j} \mathfrak{u}^{0}\right\|_{H^{3 / 2}\left(\mathbb{R}^{2}\right)}+\left\|\Gamma^{j} \mathfrak{w}^{0}\right\|_{H^{1}\left(\mathbb{R}^{2}\right)}<\infty
\end{aligned}
$$

Let

$$
\begin{aligned}
\epsilon= & \sum_{\substack{|j| \leq l+3 \\
\partial=\partial_{\alpha}, \partial_{\beta}}}\left\|\Gamma^{j}|D|^{1 / 2} z^{0}\right\|_{L^{2}\left(\mathbb{R}^{2}\right)}+\left\|\Gamma^{j} \partial z^{0}\right\|_{L^{2}\left(\mathbb{R}^{2}\right)} \\
& +\left\|\Gamma^{j} \mathfrak{u}^{0}\right\|_{H^{1 / 2}\left(\mathbb{R}^{2}\right)}+\left\|\Gamma^{j} \mathfrak{w}^{0}\right\|_{L^{2}\left(\mathbb{R}^{2}\right)} .
\end{aligned}
$$

Theorem 3.9 (3D Theorem, c.f. [35]). There exists $\epsilon_{0}>0$, such that for $0 \leq \epsilon \leq \epsilon_{0}$, the initial value problem (3.19)-(3.30) has a unique classical solution globally in time. For each time $0 \leq t<\infty$, the interface is a graph, the solution has the same regularity as the initial data and remains small. Moreover the $L^{\infty}$ norm of the steepness and the acceleration on the interface, the derivative of the velocity on the interface decay at rate $\frac{1}{t}$.

REMARK 3.10. In $[\mathbf{3 4}, \mathbf{3 5}]$ we considered only the case that the velocity $\mathbf{v} \rightarrow 0$ at the spatial infinity. One can also treat water waves with $\mathbf{v} \rightarrow c=\left(c^{\prime}, 0\right)$ a constant velocity at spatial infinity. Exact analogous computations lead to analogues of (3.8) and (3.23). This yields results like Theorems 3.8, 3.9 with $\mathbf{v} \rightarrow c$ at spatial infinity. 
REMARK 3.11. Theorems 3.8, 3.9 and their analogues for $\mathbf{v} \rightarrow c$ at spatial infinity show that there are lower bounds on the size of possible solitary waves in $2 \mathrm{D}$ and $3 \mathrm{D}$ since the $L^{\infty}$ decay of small solutions rules out small solitary waves. ${ }^{8}$

REMARK 3.12. Using the advantageous structure of the equation (3.8), $\mathrm{N}$. Totz and the author obtained a rigorous justification of the modulation approximation to the $2 \mathrm{D}$ water wave system $(1.1)(n=2)$, c.f. $[\mathbf{3 1}]$.

\section{References}

[1] D. Ambrose, N. Masmoudi The zero surface tension limit of two-dimensional water waves. Comm. Pure Appl. Math. 58 (2005), no. 10, 1287-1315.

[2] T. Beale, T. Hou \& J. Lowengrub Growth rates for the linearized motion of fluid interfaces away from equilibrium Comm. Pure Appl. Math. 46 (1993), no.9, 12691301.

[3] G. Birkhoff Helmholtz and Taylor instability Proc. Symp. in Appl. Math. Vol. XIII, pp. $55-76$.

[4] S. Chen \& Y. Zhou Decay rate of solutions to hyperbolic system of first order Acta. Math. Sinica, English series. 1999, Vol. 15, no. 4, p.471-484.

[5] D. Christodoulou, H. Lindblad On the motion of the free surface of a liquid Comm. Pure Appl. Math. 53 (2000) no. 12, 1536-1602.

[6] D. Coutand, S. Shkoller Wellposedness of the free-surface incompressible Euler equations with or without surface tension J. AMS. 20 (2007), no. 3, 829-930.

[7] R.R. Coifman, Y. Meyer Au delá des opérateurs pseudodifférentials Asterisque 57, Societé Math. de France, 1978.

[8] R. Coifman, A. McIntosh and Y. Meyer Lintegrale de Cauchy definit un operateur borne sur $L^{2}$ pour les courbes lipschitziennes, Annals of Math, 116 (1982), 361-387.

[9] R. Coifman, G. David and Y. Meyer La solution des conjectures de Calderón Adv. in Math. 48, 1983, pp. 144-148.

[10] W. Craig Non-existence of solitary water waves in three dimensions R. Soc. Lond. Philos. Trans. Ser. A Math. Phys. Eng. Sci. 360 (2002), no. 1799, 2127-2135.

[11] W. Craig An existence theory for water waves and the Boussinesq and Kortewegdevries scaling limits Comm. in P. D. E. 10(8), 1985 pp.787-1003.

[12] C.D. Crapper Introduction to water waves Ellis Horwood Ltd. 1984.

[13] P. Germain, N. Masmoudi, \& J. Shatah Global solutions of the gravity water wave equation in dimension 3 Preprint July 2009.

[14] J. Gilbert \& M. Murray Clifford algebras and Dirac operators in harmonic analysis Cambridge University Press, 1991.

[15] T. Iguchi Well-posedness of the initial value problem for capillary-gravity waves Funkcial. Ekvac. 44 (2001) no. 2, 219-241.

[16] C. Kenig Elliptic boundary value problems on Lipschitz domains Beijing Lectures in Harmonic Analysis, ed. by E. M. Stein, Princeton Univ. Press, 1986, p. 131-183.

[17] S. Klainerman The null condition and global existence to nonlinear wave equations, Lectures in Appl. Math. vol. 23 (1986), 293-325.

[18] D. Lannes Well-posedness of the water-wave equations J. Amer. Math. Soc. 18 (2005), 605-654.

[19] H. Lindblad Well-posedness for the motion of an incompressible liquid with free surface boundary Ann. of Math. 162 (2005), no. 1, 109-194.

\footnotetext{
${ }^{8}$ Non-existence of positively or negatively elevated solitary waves for 2-D infinite depth water wave with or without surface tension was established in [29]; for 3-D finite or infinite depth positively elevated water wave without surface tension it was established in [10].
} 
[20] V. I. Nalimov The Cauchy-Poisson Problem (in Russian), Dynamika Splosh. Sredy 18, 1974, pp. 104-210.

[21] M. Ogawa, A. Tani Free boundary problem for an incompressible ideal fluid with surface tension Math. Models Methods Appl. Sci. 12, (2002), no. 12, 1725-1740.

[22] C. Sogge Lectures on nonlinear wave equations International Press, 1995.

[23] G. Schneider and E. Wayne The long wave limit for the water wave problem I. The case of zero surface tension Comm. Pure. Appl. Math. 53, 2000, no. 12, 1475-1535.

[24] J. Shatah Normal forms and quadratic nonlinear Klein-Gordon equations Comm. Pure Appl. Math. 38 (1985), 685-696.

[25] J. Shatah, C. Zeng Geometry and a priori estimates for free boundary problems of the Euler's equation Comm. Pure Appl. Math. V. 61. no.5 (2008) pp. 698-744

[26] E.M.Stein Singular integrals and differentiability properties of functions Princeton University Press, 1970.

[27] W. Strauss Nonlinear wave equations CBMS No.73 AMS, 1989.

[28] Sulem, C.; Sulem, P.-L., The nonlinear Schrodinger equation. Self-focusing and wave collapse Applied Math- ematical Sciences, 139. Springer-Verlag, New York, 1999.

[29] Sun, S. M. Some analytical properties of capillary-gravity waves in two-fluid flows of infinite depth Proc. Roy. Soc. Lond. Ser.A 453 (1997) no. 1961, 1153-1175.

[30] G. I. Taylor The instability of liquid surfaces when accelerated in a direction perpendicular to their planes I. Proc. Roy. Soc. London A 201, 1950, 192-196.

[31] N. Totz \& S. Wu A Rigorous justification of the modulation approximation to the $2 D$ full water wave problem, accepted by Comm. Math. Phys.

[32] S. Wu Well-posedness in Sobolev spaces of the full water wave problem in 2-D Inventiones Mathematicae 130, 1997, pp. 39-72.

[33] S. Wu Well-posedness in Sobolev spaces of the full water wave problem in 3-D Journal of the AMS. 12. no.2 (1999), pp. 445-495.

[34] S. Wu Almost global wellposedness of the 2-D full water wave problem Inventiones Mathematicae, Vol. 177 no.1 July 2009. pp. 45-135.

[35] S. Wu Global well-posedness of the 3-D full water wave problem, Inventiones Mathematicae, Vol. 184 no.1 April 2011, pp. 125-220.

[36] H. Yosihara Gravity waves on the free surface of an incompressible perfect fluid of finite depth, RIMS Kyoto 18, 1982, pp. 49-96.

[37] P. Zhang, Z. Zhang On the free boundary problem of 3-D incompressible Euler equations. Comm. Pure. Appl. Math. V. 61 . no.7 (2008), pp. 877-940.

University of Michigan, Ann Arbor, MI 